\title{
Analyzing the genes related to Alzheimer's disease via a network and pathway-based approach
}

\author{
Yan-Shi Hu${ }^{1}$, Juncai Xin ${ }^{1}$, Ying $\mathrm{Hu}^{1}$, Lei Zhang ${ }^{2^{*}}$ and Ju Wang ${ }^{1 *}$ (D)
}

\begin{abstract}
Background: Our understanding of the molecular mechanisms underlying Alzheimer's disease (AD) remains incomplete. Previous studies have revealed that genetic factors provide a significant contribution to the pathogenesis and development of AD. In the past years, numerous genes implicated in this disease have been identified via genetic association studies on candidate genes or at the genome-wide level. However, in many cases, the roles of these genes and their interactions in $A D$ are still unclear. A comprehensive and systematic analysis focusing on the biological function and interactions of these genes in the context of AD will therefore provide valuable insights to understand the molecular features of the disease.
\end{abstract}

Method: In this study, we collected genes potentially associated with AD by screening publications on genetic association studies deposited in PubMed. The major biological themes linked with these genes were then revealed by function and biochemical pathway enrichment analysis, and the relation between the pathways was explored by pathway crosstalk analysis. Furthermore, the network features of these AD-related genes were analyzed in the context of human interactome and an AD-specific network was inferred using the Steiner minimal tree algorithm.

Results: We compiled 430 human genes reported to be associated with AD from 823 publications. Biological theme analysis indicated that the biological processes and biochemical pathways related to neurodevelopment, metabolism, cell growth and/or survival, and immunology were enriched in these genes. Pathway crosstalk analysis then revealed that the significantly enriched pathways could be grouped into three interlinked modules-neuronal and metabolic module, cell growth/survival and neuroendocrine pathway module, and immune responserelated module-indicating an AD-specific immune-endocrine-neuronal regulatory network. Furthermore, an AD-specific protein network was inferred and novel genes potentially associated with AD were identified.

Conclusion: By means of network and pathway-based methodology, we explored the pathogenetic mechanism underlying AD at a systems biology level. Results from our work could provide valuable clues for understanding the molecular mechanism underlying AD. In addition, the framework proposed in this study could be used to investigate the pathological molecular network and genes relevant to other complex diseases or phenotypes.

Keywords: Alzheimer's disease, Functional enrichment analysis, Network analysis, Pathway crosstalk

\footnotetext{
* Correspondence: Izhang@tju.edu.cn; wangju@tmu.edu.cn

${ }^{2}$ School of Computer Science and Technology, Tianjin University, Tianjin 300072, China

${ }^{1}$ School of Biomedical Engineering, Tianjin Medical University, Tianjin 300070, China
} International License (http://creativecommons.org/licenses/by/4.0/), which permits unrestricted use, distribution, and reproduction in any medium, provided you give appropriate credit to the original author(s) and the source, provide a link to the Creative Commons license, and indicate if changes were made. The Creative Commons Public Domain Dedication waiver (http://creativecommons.org/publicdomain/zero/1.0/) applies to the data made available in this article, unless otherwise stated. 


\section{Background}

Alzheimer's disease (AD) is the most prevalent neurodegenerative disorder and accounts for the majority of people diagnosed with dementia [1]. As a complex and chronic neurological disease, AD affects about 6\% of people aged 65 years and older [2], and is responsible for about 480,000 deaths per year around the world [3]. In addition to its affect on the life quality of those suffering from the disorder and their families, AD also causes a severe burden on society. In the USA alone, the health-care costs related to AD are about $\$ 172$ billion per year [4].

$\mathrm{AD}$ can be diagnosed by symptoms such as short-term memory loss, mood swings, learning impairments, and disruptions in daily activities [5]. However, as an agerelated and progressive disease, some pathological features of $\mathrm{AD}$ (e.g., amyloid deposition, accumulation of neurofibrillary tangles, as well as function and structure changes of brain regions involved in memory) often appear many years prior to clinical manifestations $[6,7]$. These pathological changes eventually lead to the damage and death of specific neurons, resulting in the emergence of clinical symptoms.

The cause of AD is still poorly understood although much effort has been dedicated to exploring the pathological and molecular mechanisms of $\mathrm{AD}$ via various approaches-e.g., animal models, gene expression profiling, genome-wide association studies (GWAS), neuroimaging techniques, or a systems biology framework [2, 8-11]. It is agreed that AD develops as a result of the combination of multiple factors, including genetic factors, a history of head injuries, depression, or hypertension. Among these factors, it is estimated that about $70 \%$ of the risk for $\mathrm{AD}$ is attributable to genetics $[1,12]$. Established genetic causes of AD include the dominant mutations of genes encoding amyloid precursor protein $(A P P)$, presenilin 1 (PSEN1), and presenilin 1 (PSEN2). However, these genes are only responsible for the pathogenesis of $\mathrm{AD}$ in about $5 \%$ of patients with clinical symptoms appearing in midlife. On the other hand, genetic analyses have suggested that, in complex disorders like $\mathrm{AD}$, individual differences can be caused by many genes and their variants. Genes with various biological functions may act in coordination to increase the risk of $\mathrm{AD}$, with a moderate or small effect exerted by each gene [1]. Consistent with this view, more and more genes-e.g., apolipoprotein E $(A P O E)$, glycogen synthase kinase 3 beta $(G S K 3 B)$, dual specificity tyrosinephosphorylation-regulated kinase $1 \mathrm{~A}(D Y R K 1 A)$, and Tau-have been found to be potentially associated with $\mathrm{AD}[1,13]$. For these genes, although a few plausible candidate genes have been partially replicated, some are considered problematic. This is especially true as highthroughput methods like GWAS are being more widely applied to genetic studies of AD. Under such circumstances, a comprehensive analysis of potential causal genes of $\mathrm{AD}$ within a pathway and/or a network framework may not only provide us with important insights beyond the conventional single-gene analyses, but also offer consolidated validation for the individual candidate gene.

In the current study, we implemented a comprehensive curation of AD-related genes from genetic association studies. We then conducted biological enrichment analyses to detect the significant functional themes within these genetic factors and analyzed the interactions among the enriched biochemical pathways by pathway crosstalk analysis. Furthermore, an AD-specific protein network was inferred and evaluated with the human protein-protein interaction network as the background. This study should offer valuable hints for understanding the molecular mechanisms of $\mathrm{AD}$ from a perspective of systems biology.

\section{Methods \\ Identification of AD-related genes}

The genes genetically associated with AD were collected by retrieving the human genetic association studies deposited in PubMed (http://www.ncbi.nlm.nih.gov/pubmed/). We retrieved publications associated with $\mathrm{AD}$ with the searching term '(Alzheimer's Disease [MeSH]) AND (Polymorphism [MeSH] OR Genotype [MeSH] OR Alleles [MeSH]) NOT (Neoplasms [MeSH])'. By July 7, 2015, a total of 5298 reports were retrieved. After reviewing all abstracts of these publications, only the genetic association studies on AD were selected. From the obtained publication pool, we then concentrated on those studies reporting a significant association of gene(s) with $\mathrm{AD}$. In order to reduce the number of potential false-positive genes, the studies reporting insignificant or negative associations were excluded even though some genes in these studies might actually be truly associated with AD. We then reviewed the full reports of each selected publication to make sure that the conclusion was consistent with its contents. In several studies, some genes were found to function cooperatively to exert significant influences on $\mathrm{AD}$, with each gene having a small or mild impact; these genes were also included in our list. In addition, the genes from several GWAS analyses on $\mathrm{AD}$, showing genetic association at a genome-wide significance level, were also included.

\section{Functional enrichment analysis of genes related to AD} WebGestalt [14] and ToppGene [15] were utilized to detect the biological themes of the AD-related genes. As a web-based bioinformation-mining platform, WebGestalt integrates information from multiple resources to determine the biological themes, including identifying the overrepresented Gene Ontology (GO) terms, amid the candidate gene listing. In this study, only the GO 
biological process terms with false discovery rate (FDR) value smaller than 0.05 were kept as the significantly enriched ones. ToppGene was used to identify and analyze the enriched biological pathways in the input genes. Pathways with FDR $<0.05$ were considered to be significantly enriched.

\section{Analysis of crosstalks among pathways}

We further built crosstalks among pathways to investigate interlinks and interactions of the enriched pathways. To measure the overlap between two pathways, the overlap coefficient $(\mathrm{OC})$ and the Jaccard coefficient (JC) were calculated using the corresponding formulas:

$$
\mathrm{OC}=\frac{|A \cap B|}{\min (|A|,|B|)}
$$

and

$$
\mathrm{JC}=\left|\frac{A \cap B}{A \cup B}\right|,
$$

in which A and B are the lists of genes of the two examined pathways. Briefly, the following procedure was adopted to construct the pathway crosstalks:

(1)Only pathways with FDR $<0.05$ were kept for crosstalk analysis. Meanwhile, pathways with five or fewer candidate genes were discarded because pathways with too few candidate genes might present few or biased connections with other pathways.

(2)Counting the common candidate genes of each pathway pair-those pathway pairs with less than two overlapped genes were removed.

(3) Measuring the overlap in every pathway pair by the corresponding JC and $\mathrm{OC}$ values.

(4) Constructing the pathway crosstalk with Cytoscape software [16].

\section{Compilation of the human protein-protein interaction network}

To explore the correlation and interaction among the ADrelated genes, we compiled a comprehensive protein-protein interaction (PPI) network, based on which the protein network topological properties of the gene set related to $\mathrm{AD}$ were calculated and analyzed. Briefly, the human protein-protein interaction data were obtained from the Protein Interaction Network Analysis (PINA) database (latest release version: May 21, 2014) [17] by pooling and curating the unique physical interaction information from six main public protein interaction databases: BioGRID, IntAct, DIP, MINT, MIPS/MPact, and HPRD. In the meantime, another interactome for Homo sapiens [18] that contained 141,296 edges (physical protein interactions) among 13,460 nodes (proteins), consisting of metabolic pathway-related interactions, regulatory and protein-protein interactions, and interaction pairs for kinase and specific substrate, was selected as an additional source of interactome data. After merging the two interactome data by excluding the selfinteracting and redundant pairs, the proteins in the list were mapped onto Entrez protein-coding genes for Homo sapiens via the Uniprot ID mapping tool (http://www.uniprot.org/uploadlists). Finally, we compiled a relatively comprehensive human physical interactome, which comprised 16,022 genes/proteins and 228,122 interactions (see Additional file 1).

\section{Construction of the AD-specific protein subnetwork}

A subnetwork specific to a given disease can provide us with hints for how the disease-related molecules interact with each other. A network parsimony principle has been demonstrated in the context of biological processes [19]; that is, the molecular networks/pathways often follow the shortest molecular paths between known disease-associated components (disease-related genes or proteins in our case). The Steiner minimal tree algorithm coincides with this biological principle, which uses a greedy heuristic strategy to iteratively link the smaller trees to larger ones until there is only one tree connecting all seed nodes [20]. GenRev [21] was utilized to identify the pathological subnetwork from the human interactome using the curated $\mathrm{AD}$-related genes as input. To assess the non-randomness of the constructed network, 1000 random networks with the same number of vertices and interactions as the $\mathrm{AD}$-specific network were generated using the Erdos-Renyi model in R igraph package [22].

\section{Results}

\section{Compilation of genes associated with AD}

Genes associated with $\mathrm{AD}$ were compiled through searching the published genetic association studies on AD in PubMed. Only the publications reporting gene(s) significantly associated with the disease were pooled, and those reporting a negative or insignificant association were excluded. Altogether, from 823 reports, we collected 430 genes reported to be associated with $\mathrm{AD}$ (Additional file 2: Table S1; the gene list is referred to as Alzgset). Among them were seven apolipoprotein genes (APOA1, APOA4, APOC1, $A P O C 2, A P O C 4, A P O D$, and $A P O E$ ), five genes encoding subunits of nicotinic acetylcholine receptors (CHRNA3, CHRNA4, CHRNA7, CHRNB2, and CHRFAM7A), four adrenoceptors (ADRA2B, ADRB1, ADRB2, and ADRB3), two serotonin receptors (HTR2A and HTR6), three dopamine degradation genes (COMT, $D B H$, and $M A O A)$, and one dopamine receptor (DRD4). A few transport-related genes were also collected, such as ATP-binding cassette transporters ( $A B C A 1, A B C A 2, A B C A 7, A B C C 2, A B C G 1$, and $A B C G 2$ ), a dopamine transporter (SLC6A3), a serotonin transporter (SLC6A4), two glucose transporters (SLC2A9 
and SLC2A14), a folate transporter (SLC19A1), and ion transporters (SLC24A4). The other genes were those involved in the biological processes related to nitric oxide synthesis (NOS1 and NOS3), immune response (e.g., IL1A, IL6, IL10, and NLRC3), as well as mitochondria-specific function (e.g., MT-ATP6, MT-CO1, MT-CYB, and MTHFR). Clearly, the genes significantly associated with $\mathrm{AD}$ were diverse in function, consistent with the complexity of this mental disorder.

\section{Biological function enrichment analysis of Alzgset}

Functional enrichment analysis revealed a more detailed biological function spectrum of these AD-related genes (see Additional file 2: Table S2). Among the GO terms overrepresented in Alzgset, those related to lipid and/or lipoprotein-related processes, drug reactions, neural development, or synaptic transmission were included. GO terms associated with drug reactions (e.g., response to ethanol, response to nicotine, and response to cocaine) and metabolic processes (e.g., xenobiotic metabolic process) were overrepresented. These results were in line with previous findings that complicated correlations existed between the pathophysiological state of $\mathrm{AD}$ and drug abuse [23, 24]. Of significance, top-ranked terms included some lipid/lipoprotein-related processes, including phospholipid efflux, reverse cholesterol transport, cholesterol homeostasis, and lipoprotein metabolic processes. Biological process terms related to synaptic transmission (e.g., positive regulation of transmission of nerve impulse; synaptic transmission, cholinergic; regulation of synaptic transmission, dopaminergic; and regulation of neurotransmitter secretion), dopamine metabolism (dopamine metabolic process), and other neural functions (e.g., synaptic vesicle transport, regulation of neuronal synaptic plasticity, neuron migration, and memory) were also enriched. Meanwhile, GO terms related to immunological function (e.g., T-helper 1 type immune response, positive regulation of interleukin- 6 production, and chronic inflammatory response) were overrepresented. The diversity in the function of ADrelated genes demonstrated the complexity of the disease.

\section{Biochemical pathway enriched in Alzgset}

Detecting the biological pathways overrepresented among Alzgset may provide useful information about the pathogenic molecular mechanism underlying AD. For Alzgset, 68 enriched pathways were identified (Table 1). Among them, several pathways related to immune processes were included (e.g., cytokines and inflammatory response, cytokine network, dendritic cells in regulating $\mathrm{TH} 1$ and $\mathrm{TH} 2$ development, and $\mathrm{IL}-5$ signaling), consistent with previous studies [25, 26]. Also, neurotransmitter signaling-related pathways were identified, such as cholinergic synapse, dopaminergic synapse, serotonergic synapse, and so forth. Additionally, in the Alzgset enriched pathway list, there were some pathways related to cell growth and/or survival, including neurotrophin signaling, PI3K-Akt signaling, mTOR signaling, Notch signaling, and so forth, which are vital for cell growth/survival state of neurons in the process of $\mathrm{AD}[27,28]$. Moreover, metabolism-related pathways, consisting of drug metabolism (cytochrome P450), glutathione metabolism, and metabolism of xenobiotics by cytochrome P450, were also significantly enriched, indicating that related metabolism processes were involved in the etiology and development processes of $\mathrm{AD}$. What is more, the pathway of the intestinal immune network for IgA production was enriched, which might suggest a connection between $\mathrm{AD}$ and the intestinal microbiota $[29,30]$. Furthermore, pathways involved in osteoclast differentiation and adipocytokine signaling were also detected, complying with prior studies [31-33].

\section{Crosstalks among significantly enriched pathways}

To explore the correlations between the pathways, we implemented a pathway crosstalk analysis for the 68 enriched pathways. Here we assumed that crosstalk existed in a pathway pair if they had a proportion of common genes in Alzgset [34]. There were 41 pathways including six or more members in Alzgset, of which 37 pathways met the criterion for crosstalk analysis; that is, each pathway shared at least two genes with one or more other pathways. All of the pathway pairs (207 crosstalks among 37 pathways) were used for constructing the pathway crosstalk network and the overlap significance of each pathway pair was evaluated based on the average of JC and OC.

Based on their crosstalks, these pathways could be roughly divided into three major modules, with pathways in each group having more crosstalks with each other than with those outside of this module and more likely being related to the same or similar biological process (Fig. 1). The first module primarily included neuronal-related and xenobiotic or drug metabolismrelated pathways (e.g., calcium signaling, dopaminergic synapse, cholinergic synapse, serotonergic synapse and neurotrophin signaling, metabolism of xenobiotics by cytochrome $\mathrm{P} 450$, and drug metabolism-cytochrome P450). The major theme of the second module was cell growth/survival and neuroendocrine-related pathways (e.g., PI3K-Akt signaling, mTOR signaling, notch signaling, prolactin signaling, etc.). The third module included immune response-related pathways (e.g., toll-like receptor signaling, Fc epsilon RI signaling pathway). At the same time, the three modules were interlinked with each other, indicating the existence of an AD-specific immune-endocrine-neuronal regulatory network. 
Table 1 Pathways enriched in Alzgset ${ }^{\mathrm{a}}$

\begin{tabular}{|c|c|c|c|}
\hline Pathway & $p$ value $^{\text {b }}$ & $p_{\mathrm{BH}}$ value $^{\mathrm{c}}$ & Genes included in the pathway ${ }^{d}$ \\
\hline Cytokines and inflammatory response & $1.03 \times 10^{-9}$ & $8.79 \times 10^{-8}$ & $\begin{array}{l}\text { CXCL8, HLA-DRA, HLA-DRB1, IL10, IL12A, IL12B, IL1A, IL4, } \\
I L 6, T G F B 1, T N F\end{array}$ \\
\hline cytokine network & $9.89 \times 10^{-9}$ & $3.84 \times 10^{-7}$ & CXCL8, IL10, IL12A, IL12B, IL18, IL1A, IL4, IL6, TNF \\
\hline Hematopoietic cell lineage & $1.92 \times 10^{-7}$ & $5.46 \times 10^{-6}$ & $\begin{array}{l}\text { CD14, CD33, CD36, CD44, CR1, HLA-DRA, HLA-DRB1, } \\
\text { HLA-DRB5, IL1A, IL1B, IL4, IL6, IL6R, MME, TNF }\end{array}$ \\
\hline $\begin{array}{l}\text { Dendritic cells in regulating } \mathrm{TH} 1 \text { and } \mathrm{TH} 2 \\
\text { Development }\end{array}$ & $3.11 \times 10^{-7}$ & $8.29 \times 10^{-6}$ & CD33, IL10, IL12A, IL12B, IL4, TLR2, TLR4, TLR9 \\
\hline Ovarian steroidogenesis & $5.88 \times 10^{-6}$ & $1.09 \times 10^{-4}$ & $\begin{array}{l}\text { ALOX5, CYP19A1, FSHR, IGF1, INS, LDLR, LHCGR, PLA2G4A, } \\
\text { PTGS2, STAR }\end{array}$ \\
\hline IL-5 signaling pathway & $9.00 \times 10^{-6}$ & $1.60 \times 10^{-4}$ & HLA-DRA, HLA-DRB1, IL1B, IL4, IL6 \\
\hline Neurotrophin signaling pathway & $1.08 \times 10^{-5}$ & $1.77 \times 10^{-4}$ & $\begin{array}{l}\text { BDNF, CAMK2D, GSK3B, IRS1, NGF, NGFR, NTF3, NTRK1, } \\
\text { NTRK2, PIK3R1, PSEN1, PSEN2, SOS2, TP53, TP73 }\end{array}$ \\
\hline HIF-1 signaling pathway & $1.12 \times 10^{-5}$ & $1.77 \times 10^{-4}$ & $\begin{array}{l}\text { CAMK2D, EIF4EBP1, GAPDH, HMOX1, IGF1, IL6, IL6R, INS, } \\
\text { NOS3, PIK3R1, RPS6KB2, TF, TLR4, VEGFA }\end{array}$ \\
\hline NOD-like receptor signaling pathway & $1.66 \times 10^{-5}$ & $2.37 \times 10^{-4}$ & $\begin{array}{l}\text { CARD8, CCL2, CXCL8, IL18, IL1B, IL6, MEFV, NLRP1, } \\
\text { NLRP3, TNF }\end{array}$ \\
\hline $\begin{array}{l}\text { Mechanism of gene regulation by peroxisome } \\
\text { proliferators via PPARa }\end{array}$ & $1.95 \times 10^{-5}$ & $2.69 \times 10^{-4}$ & $\begin{array}{l}\text { APOA1, CD36, INS, LPL, PIK3R1, PPARA, PTGS2, RXRA, } \\
\text { SP1, TNF }\end{array}$ \\
\hline Th1/Th2 differentiation & $2.54 \times 10^{-5}$ & $3.19 \times 10^{-4}$ & HLA-DRA, HLA-DRB1, IL12A, IL12B, IL18, IL4 \\
\hline Antigen-dependent B-cell activation & $2.68 \times 10^{-5}$ & $3.26 \times 10^{-4}$ & FAS, HLA-DRA, HLA-DRB1, IL10, IL4 \\
\hline Oxidative phosphorylation & $3.74 \times 10^{-5}$ & $4.39 \times 10^{-4}$ & $\begin{array}{l}\text { COX10, COX15, MT-ATP6, MT-ATP8, MT-CO1, MT-CO2, } \\
\text { MT-CO3, MT-CYB, MT-ND1, MT-ND2, MT-ND3, MT-ND4, } \\
\text { MT-ND4L, MT-ND5, MT-ND6 }\end{array}$ \\
\hline PI3K-Akt signaling pathway & $3.80 \times 10^{-5}$ & $4.39 \times 10^{-4}$ & $\begin{array}{l}\text { COL11A1, EFNA5, EIF4EBP1, FGF1, GNB3, GSK3B, IGF1, IL4, } \\
\text { IL6, IL6R, INS, IRS1, NGF, NGFR, NOS3, PCK1, PIK3R1, } \\
\text { PPP2R2B, RELN, RPS6KB2, RXRA, SOS2, TLR2, TLR4, TP53, } \\
\text { VEGFA, YWHAQ }\end{array}$ \\
\hline NF-kB signaling pathway & $4.83 \times 10^{-5}$ & $5.42 \times 10^{-4}$ & $\begin{array}{l}\text { CD14, CXCL8, ICAM1, IL1B, LCK, PARP1, PLAU, PTGS2, TLR4, } \\
\text { TNF, TRAF2, UBE2I }\end{array}$ \\
\hline Phagosome & $7.77 \times 10^{-5}$ & $8.29 \times 10^{-4}$ & $\begin{array}{l}\text { CD14, CD36, CTSS, HLA-A, HLA-DQB1, HLA-DRA, HLA-DRB1, } \\
\text { HLA-DRB5, MBL2, MPO, NOS1, OLR1, RABTA, TAP2, TLR2, TLR4 }\end{array}$ \\
\hline Erythrocyte differentiation pathway & $9.33 \times 10^{-5}$ & $9.49 \times 10^{-4}$ & $C C L 3, I G F 1, I L 1 A, I L 6, T G F B 1$ \\
\hline IL-10 anti-inflammatory signaling pathway & $1.82 \times 10^{-4}$ & $1.69 \times 10^{-3}$ & HMOX1, IL10, ILIA, IL6, TNF \\
\hline $\begin{array}{l}\text { Cells and molecules involved in local acute } \\
\text { inflammatory response }\end{array}$ & $1.82 \times 10^{-4}$ & $1.69 \times 10^{-3}$ & CXCL8, ICAM1, ILIA, IL6, TNF \\
\hline Toll-like receptor signaling pathway & $2.15 \times 10^{-4}$ & $1.95 \times 10^{-3}$ & $\begin{array}{l}\text { CCL3, CD14, CXCL8, IL12A, IL12B, IL1B, IL6, PIK3R1, TLR2, } \\
\text { TLR4, TLR9, TNF }\end{array}$ \\
\hline Free radical induced apoptosis & $2.22 \times 10^{-4}$ & $1.97 \times 10^{-3}$ & CXCL8, GPX1, SOD1, TNF \\
\hline Intestinal immune network for IgA production & $2.65 \times 10^{-4}$ & $2.26 \times 10^{-3}$ & $\begin{array}{l}\text { HLA-DQB1, HLA-DRA, HLA-DRB1, HLA-DRB5, IL10, IL4, IL6, } \\
\text { TGFB1 }\end{array}$ \\
\hline $\begin{array}{l}\text { Selective expression of chemokine receptors } \\
\text { during T-cell polarization }\end{array}$ & $3.35 \times 10^{-4}$ & $2.68 \times 10^{-3}$ & CCL3, CCR2, IL12A, IL12B, IL4, TGFB1 \\
\hline B lymphocyte cell surface molecules & $3.39 \times 10^{-4}$ & $2.68 \times 10^{-3}$ & CR1, HLA-DRA, HLA-DRB1, ICAM1 \\
\hline $\begin{array}{l}\text { Phosphorylation of MEK1 by cdk5/p35 } \\
\text { downregulates the MAP kinase pathway }\end{array}$ & $3.39 \times 10^{-4}$ & $2.68 \times 10^{-3}$ & CDK5, CDK5R1, NGF, NGFR \\
\hline Complement and coagulation cascades & $4.61 \times 10^{-4}$ & $3.58 \times 10^{-3}$ & $\begin{array}{l}\text { A2M, C4A, C4B, CFH, CR1, F13A1, MBL2, PLAU, } \\
\text { SERPINA1 }\end{array}$ \\
\hline$A B C$ transporters & $5.87 \times 10^{-4}$ & $4.32 \times 10^{-3}$ & $A B C A 1, A B C A 2, A B C A 7, A B C C 2, A B C G 1, A B C G 2, T A P 2$ \\
\hline Signal transduction through IL-1R & $6.97 \times 10^{-4}$ & $5.05 \times 10^{-3}$ & ILIA, ILIB, ILIRN, IL6, TGFB1, TNF \\
\hline mTOR signaling pathway & $8.19 \times 10^{-4}$ & $5.83 \times 10^{-3}$ & $\begin{array}{l}\text { EIF4EBP1, IGF1, INS, IRS1, PIK3R1, RPS6KB2, TNF, } \\
\text { VEGFA }\end{array}$ \\
\hline Adhesion and diapedesis of granulocytes & $9.49 \times 10^{-4}$ & $6.65 \times 10^{-3}$ & CXCL8, ICAM1, ILIA, TNF \\
\hline
\end{tabular}


Table 1 Pathways enriched in Alzgset ${ }^{\mathrm{a}}$ (Continued)

\begin{tabular}{|c|c|c|c|}
\hline TNF signaling pathway & $1.12 \times 10^{-3}$ & $7.69 \times 10^{-3}$ & $\begin{array}{l}\text { CCL2, FAS, ICAM1, IL1B, IL6, MAGI2, MMP3, PIK3R1, } \\
\text { PTGS2, TNF, TRAF2 }\end{array}$ \\
\hline MAPK signaling pathway & $1.13 \times 10^{-3}$ & $7.69 \times 10^{-3}$ & $\begin{array}{l}\text { BDNF, CD14, FAS, FGF1, IL1A, IL1B, MAPK8IP1, MAPT, } \\
\text { MEF2C, NGF, NTF3, NTRK1, NTRK2, PLA2G4A, SOS2, } \\
\text { TGFB1, TNF, TP53, TRAF2 }\end{array}$ \\
\hline The IGF-1 receptor and longevity & $1.26 \times 10^{-3}$ & $8.28 \times 10^{-3}$ & IGF1, PIK3R1, SOD1, SOD2 \\
\hline Glutathione metabolism & $1.45 \times 10^{-3}$ & $8.95 \times 10^{-3}$ & GPX1, GSTM1, GSTM3, GSTO1, GSTO2, GSTP1, GSTT1 \\
\hline Cytokine-cytokine receptor interaction & $1.48 \times 10^{-3}$ & $8.95 \times 10^{-3}$ & $\begin{array}{l}C C L 2, C C L 3, C C R 2, C X C L 8, F A S, I L 10, I L 12 A, I L 12 B, I L 18, \\
I L 1 A, I L 1 B, I L 23 R, I L 4, I L 6, I L 6 R, N G F R, T G F B 1, T N F, V E G F A\end{array}$ \\
\hline Serotonergic synapse & $1.50 \times 10^{-3}$ & $8.95 \times 10^{-3}$ & $\begin{array}{l}\text { ALOX5, APP, CYP2D6, GNB3, HTR2A, HTR6, KCNJ6, } \\
\text { MAOA, PLA2G4A, PTGS2, SLC6A4 }\end{array}$ \\
\hline Antigen processing and presentation & $1.63 \times 10^{-3}$ & $9.53 \times 10^{-3}$ & $\begin{array}{l}\text { CTSS, HLA-A, HLA-DQB1, HLA-DRA, HLA-DRB1, } \\
\text { HLA-DRB5, HSPA5, TAP2, TNF }\end{array}$ \\
\hline Drug metabolism—cytochrome P450 & $1.88 \times 10^{-3}$ & $1.05 \times 10^{-2}$ & $\begin{array}{l}\text { CYP2D6, GSTM1, GSTM3, GSTO1, GSTO2, GSTP1, } \\
\text { GSTT1, MAOA }\end{array}$ \\
\hline Cell cycle: G1/S check point & $2.13 \times 10^{-3}$ & $1.18 \times 10^{-2}$ & CDK1, CDKN2A, GSK3B, TGFB1, TP53 \\
\hline Fсع RI signaling pathway & $2.26 \times 10^{-3}$ & $1.23 \times 10^{-2}$ & $\begin{array}{l}\text { FCER1G, GAB2, IL4, INPP5D, PIK3R1, PLA2G4A, } \\
\text { SOS2, TNF }\end{array}$ \\
\hline Apoptosis & $2.28 \times 10^{-3}$ & $1.23 \times 10^{-2}$ & $\begin{array}{l}\text { FAS, IL1A, IL1B, NGF, NTRK1, PIK3R1, TNF, TP53, } \\
\text { TRAF2 }\end{array}$ \\
\hline Role of Erk5 in neuronal survival & $2.61 \times 10^{-3}$ & $1.39 \times 10^{-2}$ & MEF2A, MEF2C, NTRK1, PIK3R1 \\
\hline Bioactive peptide-induced signaling pathway & $2.90 \times 10^{-3}$ & $1.52 \times 10^{-2}$ & CAMK2D, CDK5, GNA11, MAPT, MYLK, PTK2B \\
\hline $\begin{array}{l}\text { Control of skeletal myogenesis by HDAC and } \\
\text { calcium/calmodulin-dependent kinase (CaMK) }\end{array}$ & $2.93 \times 10^{-3}$ & $1.52 \times 10^{-2}$ & IGFI, INS, MEF2A, MEF2C, PIK3R1 \\
\hline Metabolism of xenobiotics by cytochrome P450 & $3.22 \times 10^{-3}$ & $1.62 \times 10^{-2}$ & $\begin{array}{l}\text { CYP2D6, GSTM1, GSTM3, GSTO1, GSTO2, GSTP1, } \\
\text { GSTT1, HSD11B1 }\end{array}$ \\
\hline $\begin{array}{l}\text { Ras-independent pathway in NK cell-mediated } \\
\text { cytotoxicity }\end{array}$ & $3.92 \times 10^{-3}$ & $1.88 \times 10^{-2}$ & HLA-A, IL18, PIK3R1, PTK2B \\
\hline Dopaminergic synapse & $4.48 \times 10^{-3}$ & $2.11 \times 10^{-2}$ & $\begin{array}{l}\text { CAMK2D, CLOCK, COMT, DRD4, GNB3, GRIN2B, } \\
\text { GSK3B, KCNJ6, MAOA, PPP2R2B, SLC6A3 }\end{array}$ \\
\hline Cholinergic synapse & $4.57 \times 10^{-3}$ & $2.12 \times 10^{-2}$ & $\begin{array}{l}\text { CAMK2D, CHAT, CHRNA3, CHRNA4, CHRNA7, } \\
\text { CHRNB2, GNA11, GNB3, KCNJ6, PIK3R1 }\end{array}$ \\
\hline The co-stimulatory signal during T-cell activation & $4.72 \times 10^{-3}$ & $2.17 \times 10^{-2}$ & HLA-DRA, HLA-DRB1, LCK, PIK3R1 \\
\hline Adhesion and diapedesis of lymphocytes & $5.03 \times 10^{-3}$ & $2.28 \times 10^{-2}$ & CXCL8, ICAM1, ILIA \\
\hline Notch signaling pathway & $5.07 \times 10^{-3}$ & $2.28 \times 10^{-2}$ & APH1A, APH1B, NCSTN, PSEN1, PSEN2, PSENEN \\
\hline Role of ERBB2 in signal transduction and oncology & $5.61 \times 10^{-3}$ & $2.50 \times 10^{-2}$ & ESR1, IL6, IL6R, PIK3R1 \\
\hline Aminoacyl-tRNA biosynthesis & $6.37 \times 10^{-3}$ & $2.80 \times 10^{-2}$ & MT-TG, MT-TH, MT-TL2, MT-TQ, MT-TR, MT-TS2, MT-TT \\
\hline Trka receptor signaling pathway & $6.55 \times 10^{-3}$ & $2.80 \times 10^{-2}$ & NGF, NTRK1, PIK3R1 \\
\hline Rac 1 cell motility signaling pathway & $6.62 \times 10^{-3}$ & $2.80 \times 10^{-2}$ & CDK5, CDK5R1, MYLK, PIK3R1 \\
\hline CTCF: first multivalent nuclear factor & $6.62 \times 10^{-3}$ & $2.80 \times 10^{-2}$ & CDKN2A, PIK3R1, TGFB1, TP53 \\
\hline Regulation of PGC-1a & $7.74 \times 10^{-3}$ & $3.21 \times 10^{-2}$ & CAMK2D, MEF2A, MEF2C, PPARA \\
\hline Calcium signaling pathway & $7.85 \times 10^{-3}$ & $3.22 \times 10^{-2}$ & $\begin{array}{l}\text { ADRB1, ADRB2, ADRB3, CAMK2D, CHRNA7, GNA11, } \\
\text { HTR2A, HTR6, LHCGR, MYLK, NOS1, NOS3, PTK2B }\end{array}$ \\
\hline $\begin{array}{l}\text { LCk and Fyn tyrosine kinases in initiation of } \\
\text { TCR activation }\end{array}$ & $8.30 \times 10^{-3}$ & $3.38 \times 10^{-2}$ & HLA-DRA, HLA-DRB1, LCK \\
\hline Adipocytokine signaling pathway & $8.75 \times 10^{-3}$ & $3.52 \times 10^{-2}$ & CD36, IRS1, PCK1, PPARA, RXRA, TNF, TRAF2 \\
\hline Ras signaling pathway & $9.43 \times 10^{-3}$ & $3.76 \times 10^{-2}$ & $\begin{array}{l}\text { EFNA5, EXOC2, FGF1, GAB2, GNB3, GRIN2B, IGF1, } \\
\text { INS, NGF, NGFR, PIK3R1, PLA2G3, PLA2G4A, SOS2, } \\
\text { VEGFA }\end{array}$ \\
\hline Prolactin signaling pathway & $1.02 \times 10^{-2}$ & $3.96 \times 10^{-2}$ & ESR1, ESR2, GSK3B, INS, LHCGR, PIK3R1, SOS2 \\
\hline Catecholamine biosynthesis, tyrosine $\rightarrow$ & $1.05 \times 10^{-2}$ & $3.99 \times 10^{-2}$ & $D B H, P N M T$ \\
\hline
\end{tabular}


Table 1 Pathways enriched in Alzgset ${ }^{\mathrm{a}}$ (Continued)

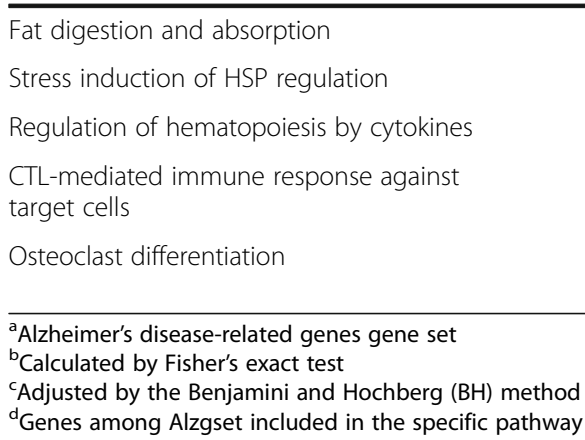

\section{AD-specific protein network}

To further examine the potential pathological protein network of Alzgset, we constructed a subnetwork for $\mathrm{AD}$ from the human protein-protein interaction network via the Steiner minimal tree algorithm. This method tries to connect the largest number of input nodes (genes included in Alzgset in our case) via the least number of interlinking nodes. As shown in Fig. 2, the protein network of AD comprised 496 nodes and 1521 edges (interactions).

As shown, 393 out of 430 Alzgset genes were included in the AD-specific network, which accounted for $79.2 \%$ of 496 genes in the network and $91.4 \%$ of Alzgset, demonstrating a high coverage of Alzgset in the subnetwork. There were 103 genes in the AD-specific molecular network outside of Alzgset (Table 2). Given that these intermediate genes interacted closely with those known to be related to $\mathrm{AD}$, they might also be involved in the pathological process of the disease phenotype. Notably, a number of the genes-e.g., epidermal growth factor receptor $(E G F R)$, nuclear respiratory factor 1 (NRF1), somatostatin receptor 2 (SSTR2), and sortilin 1 (SORT1) - had been shown related to AD in several previous studies [35-38]. Some of these genes have not been reported to be directly involved in the pathophysiological condition of $\mathrm{AD}$, but genes linking to them or other members of the same protein family may have been found to play a role in such processes. For instance, ATP binding cassette subfamily G member 5 (ABCG5), a member of a transport system superfamily, involved in ATP binding and transporting of substrates across cytomembranes, was a node in the AD-specific network

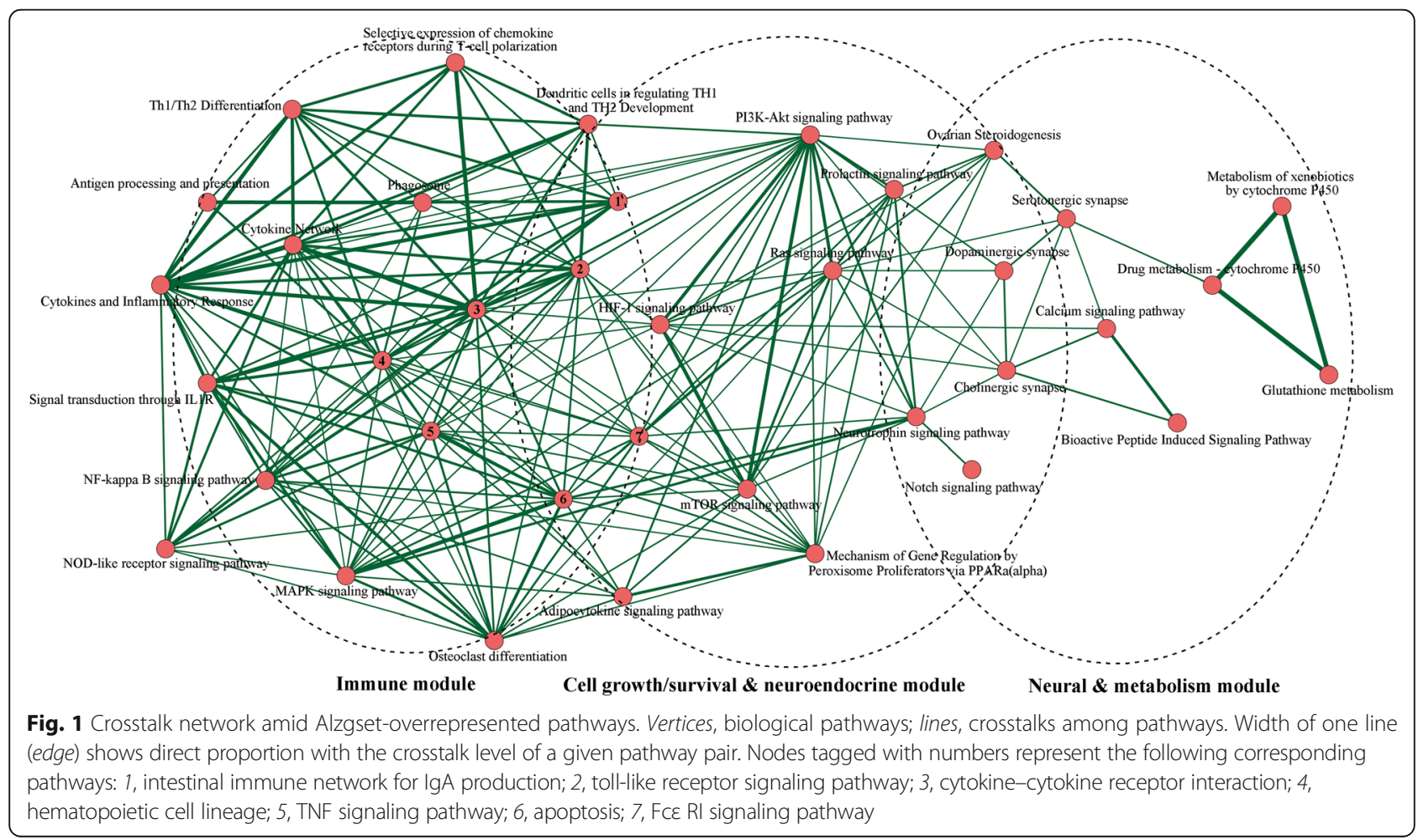




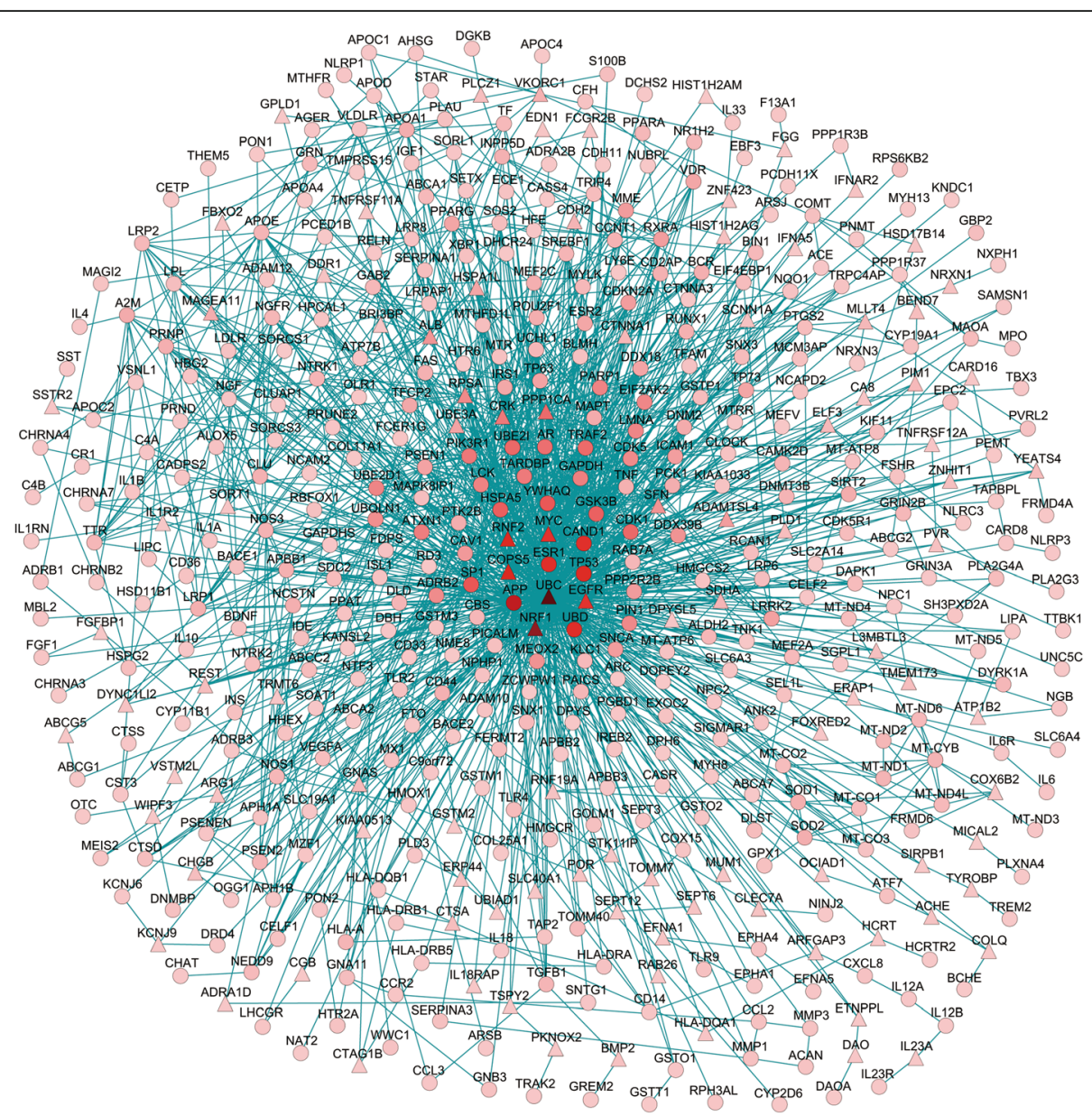

Fig. 2 AD-specific protein network built by means of the Steiner minimal tree algorithm, including 496 vertices and 1521 lines. Circular vertices, genes of Alzgset; triangular vertices, expanding genes. Color of a typical vertex designates its corresponding degree under the background of the human protein interactome. Darkness of color for a vertex is directly proportional to the corresponding degree value (Color figure online)

but was out of Alzgset. However, six members from the same family were included in Alzgset $(A B C A 1$, $A B C A 2, A B C A 7, A B C C 2, A B C G 1$, and $A B C G 2)$, and there was experimental evidence for their involvement in $\mathrm{AD}$; for example, the expression reduction or loss of function of $A B C A 7$ could alter Alzheimer amyloid processing [39]. Solute carrier family 40 member 1 (SLC40A1), encoding a cytomembrane protein that may be linked to iron export from duodenal epithelial cells, was also included in the AD-specific network. SLC40A1can interact with Golgi membrane protein 1 (GOLM1) and hepcidin antimicrobial peptide $(H A M P)$. The former was a gene in Alzgset and its mutation may be related to reduced regional gray matter volume in $\mathrm{AD}$ patients [40], and the expression of HAMP was significantly reduced in hippocampal lysates from AD brains [41]. Thus, it is likely that some of the 103 genes in the AD-specific network may play roles in $\mathrm{AD}$ susceptibility and can be novel targets for further exploration.

\section{Discussion}

We have made great progress in exploring the molecular mechanisms of Alzheimer's disease in recent years. With the advancement and maturity of high-throughput technology, we are able to identify the elements related to this disease on much larger scales. Although more and more genes/proteins potentially involved in the disease have been reported, a thorough analysis of the biochemical processes associated with the pathogenesis of $\mathrm{AD}$ from the molecular aspect is still missing. In such cases, a systematic analysis of ADrelated genes via a pathway-based and network-based analytical framework will provide us with insight into the disease beyond the single candidate gene-based analyses [42-44]. In this study, by pooling and curating human genes related to AD from genetic studies, and systematically delineating the interconnection of these genes by means of pathway-based and networkbased analyses, we analyzed AD-related biochemical processes and their interactions. 
Table 2 Genes included in the AD-specific network but not in Alzgset $^{a}$

\begin{tabular}{|c|c|c|c|}
\hline \multicolumn{2}{|l|}{ Alzgsel } & \multicolumn{2}{|c|}{ Alzgsel (conninuea) } \\
\hline Gene symbol & Gene name & GSTM2 & Glutathione S-transferase mu 2 (muscle) \\
\hline$A B C G 5$ & ATP binding cassette subfamily $\mathrm{G}$ member 5 & HCRT & Hypocretin (orexin) neuropeptide precursor \\
\hline ACHE & Acetylcholinesterase (Yt blood group) & HIST1H2AG & Histone cluster 1, H2ag \\
\hline ADAMTSL4 & ADAMTS-like 4 & HIST1H2AM & Histone cluster 1, H2am \\
\hline ADRA1D & Adrenoceptor alpha 1D & HLA-DQA1 & Major histocompatibility complex, class II, DQ alpha 1 \\
\hline$A L B$ & Albumin & HSD17B14 & Hydroxysteroid (17-beta) dehydrogenase 14 \\
\hline ARFGAP3 & ADP-ribosylation factor GTPase activating protein 3 & HSPA1L & Heat shock $70 \mathrm{kDa}$ protein 1-like \\
\hline ARG1 & Arginase 1 & IFNA5 & Interferon, alpha 5 \\
\hline ATP1B2 & ATPase, $\mathrm{Na}^{+} / \mathrm{K}^{+}$transporting, beta 2 polypeptide & IFNAR2 & Interferon (alpha, beta and omega) receptor 2 \\
\hline BEND7 & BEN domain containing 7 & IL 18RAP & Interleukin-18 receptor accessory protein \\
\hline BMP2 & Bone morphogenetic protein 2 & $\operatorname{lL} 1 R 2$ & Interleukin-1 receptor, type II \\
\hline$B R I 3 B P$ & BRI3 binding protein & IL23A & Interleukin-23, alpha subunit p19 \\
\hline CA8 & Carbonic anhydrase VIII & KCNJ9 & Potassium channel, inwardly rectifying subfamily J, \\
\hline CARD16 & Caspase recruitment domain family, member 16 & & \\
\hline $\mathrm{CDH} 2$ & Cadherin 2, type 1, N-cadherin (neuronal) & KIAA0513 & KIAA0513 \\
\hline$C G B$ & Chorionic gonadotropin, beta polypeptide & L3MBTL3 & L(3)mbt-like 3 (Drosophila) \\
\hline CHGB & Chromogranin B & MAGEA11 & Melanoma antigen family A11 \\
\hline CLECTA & C-type lectin domain family 7 , member A & MICAL2 & $\begin{array}{l}\text { Microtubule associated monooxygenase, calponin } \\
\text { and LIM domain containing } 2\end{array}$ \\
\hline COLQ & $\begin{array}{l}\text { Collagen-like tail subunit (single strand of homotrimer) } \\
\text { of asymmetric acetylcholinesterase }\end{array}$ & MLLT4 & $\begin{array}{l}\text { Myeloid/lymphoid or mixed-lineage leukemia; } \\
\text { translocated to, } 4\end{array}$ \\
\hline COPS5 & COP9 signalosome subunit 5 & MUM1 & Melanoma associated antigen (mutated) 1 \\
\hline COX6B2 & Cytochrome c oxidase subunit VIb polypeptide 2 (testis) & MYC & V-myc avian myelocytomatosis viral oncogene \\
\hline CRK & V-crk avian sarcoma virus CT10 oncogene homolog & & homolog \\
\hline CTAGIB & Cancer/testis antigen 1B & NRF1 & Nuclear respiratory factor 1 \\
\hline CTNNA1 & Catenin (cadherin-associated protein), alpha 1, $102 \mathrm{kDa}$ & NRXN1 & Neurexin 1 \\
\hline CTSA & Cathepsin A & OCIAD1 & OCIA domain containing 1 \\
\hline DAO & D-amino-acid oxidase & PIM1 & Pim-1 proto-oncogene, serine/threonine kinase \\
\hline DDR1 & Discoidin domain receptor tyrosine kinase 1 & PKNOX2 & PBX/knotted 1 homeobox 2 \\
\hline DPYSL5 & Dihydropyrimidinase-like 5 & PLCZ1 & Phospholipase C, zeta 1 \\
\hline DYNC1LI2 & Dynein, cytoplasmic 1, light intermediate chain 2 & PLD1 & Phospholipase D1, phosphatidylcholine-specific \\
\hline EDN1 & Endothelin 1 & $P O R$ & P450 (cytochrome) oxidoreductase \\
\hline EFNA1 & Ephrin-A1 & PPPICA & Protein phosphatase 1, catalytic subunit, alpha \\
\hline EGFR & Epidermal growth factor receptor & & \\
\hline ELF3 & E74-like factor 3 (ets domain transcription factor, & $P V R$ & Poliovirus receptor \\
\hline & epithelial-specific) & $R A B 26$ & RAB26, member RAS oncogene family \\
\hline ERAP1 & Endoplasmic reticulum aminopeptidase 1 & REST & RE1-silencing transcription factor \\
\hline ERP44 & Endoplasmic reticulum protein 44 & RNF19A & Ring finger protein 19A, RBR E3 ubiquitin protein \\
\hline ETNPPL & Ethanolamine-phosphate phospho-lyase & & ligase \\
\hline$F B X O 2$ & F-box protein 2 & RNF2 & Ring finger protein 2 \\
\hline FCGR2B & Fc fragment of IgG, low affinity Ilb, receptor (CD32) & RPSA & Ribosomal protein SA \\
\hline FGFBP1 & Fibroblast growth factor binding protein 1 & SCNN1A & Sodium channel, non voltage gated 1 alpha subunit \\
\hline FGG & Fibrinogen gamma chain & SDHA & $\begin{array}{l}\text { Succinate dehydrogenase complex, subunit } \mathrm{A}, \\
\text { flavoprotein ( } \mathrm{Fp} \text { ) }\end{array}$ \\
\hline FOXRED2 & FAD-dependent oxidoreductase domain containing 2 & SEPT12 & 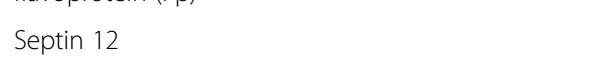 \\
\hline GNAS & GNAS complex locus & SEPT6 & Septin 6 \\
\hline GPLD1 & Glycosylphosphatidylinositol specific phospholipase D1 & SFN & Stratifin \\
\hline
\end{tabular}

Table 2 Genes included in the AD-specific network but not in Alzgset $^{\mathrm{a}}$ (Continued) 
Table 2 Genes included in the AD-specific network but not in Alzgset $^{\mathrm{a}}$ (Continued)

\begin{tabular}{|c|c|}
\hline SIRPB1 & Signal-regulatory protein beta 1 \\
\hline SLC4OA1 & Solute carrier family 40 member 1 \\
\hline SORT1 & Sortilin 1 \\
\hline SSTR2 & Somatostatin receptor 2 \\
\hline STK11IP & Serine/threonine kinase 11 interacting protein \\
\hline TMEM173 & Transmembrane protein 173 \\
\hline TNFRSF11A & $\begin{array}{l}\text { Tumor necrosis factor receptor superfamily, } \\
\text { member } 11 \text { a, NFKB activator }\end{array}$ \\
\hline TNFRSF12A & $\begin{array}{l}\text { Tumor necrosis factor receptor superfamily, } \\
\text { member } 12 \mathrm{~A}\end{array}$ \\
\hline TOMM7 & $\begin{array}{l}\text { Translocase of outer mitochondrial membrane } \\
7 \text { homolog (yeast) }\end{array}$ \\
\hline TRMT6 & tRNA methyltransferase 6 \\
\hline TSPY2 & Testis specific protein, Y-linked 2 \\
\hline TYROBP & TYRO protein tyrosine kinase binding protein \\
\hline$U B C$ & Ubiquitin C \\
\hline UBE3A & Ubiquitin protein ligase $\mathrm{E} 3 \mathrm{~A}$ \\
\hline UBIAD1 & UbiA prenyltransferase domain containing 1 \\
\hline VKORC1 & Vitamin K epoxide reductase complex, subunit 1 \\
\hline VSTM2L & V-set and transmembrane domain containing 2 like \\
\hline WIPF3 & WAS/WASL interacting protein family, member 3 \\
\hline YEATS4 & YEATS domain containing 4 \\
\hline ZNF423 & Zinc finger protein 423 \\
\hline ZNHIT1 & Zinc finger, HIT-type containing 1 \\
\hline
\end{tabular}

$A D$ Alzheimer's disease

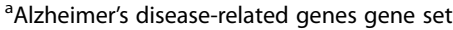

Compared with the candidate gene(s)-based approach, a comprehensive analysis on AD-related genes conducted in this study has its own advantages. By implementing an extensive compilation and curation of human genes from genetic association studies on AD, we could obtain valuable gene source data for further analysis. Especially, because the risk of AD susceptibility can be attributed to many genes, with multiple genes functioning in a concerted manner and each gene exerting a small effect [45], we took this into consideration by also retrieving genes jointly showing significant genetic association with AD. At the same time, by focusing on the biological correlation of genes, pathway and network analysis can not only give us a more comprehensive view for the pathological mechanisms of $\mathrm{AD}$, but are also more robust to the influence of false-positive genes.

As revealed by function enrichment analysis, genes in Alzgset may play important roles in lipid/lipoprotein-related procedures, the immune system, the metabolic process, drug response processes, and neurodevelopment. For example, terms such as reverse cholesterol transport, positive regulation of interleukin-6 production, response to ethanol, lipoprotein metabolic process, diol metabolic process, xenobiotic metabolic process, and regulation of neuronal synaptic plasticity were overrepresented among Alzgset genes, implying the important roles of these processes in the pathological processes of $\mathrm{AD}$. Furthermore, we noticed several terms of memory, visual learning, social behavior, sleep, axon regeneration, and axon guidance also emerged in the enriched list, concurrent with a-priori biological findings for AD [46-50].

Our biochemical pathway analysis showed that immune-related pathways were enriched among Alzgset, which further highlighted the connections between $\mathrm{AD}$ and immune-related biological activities. Previous studies have shown the involvement of neuroinflammation in AD pathology, with inflammatory cytokines exerting central efforts [51, 52]. Simultaneously, four pathways associated with neurotransmitters were found to be overrepresented in Alzgset, coinciding with their essential roles in the etiology and progression of $\mathrm{AD}$. Acetylcholine, dopamine, and serotonin are major neurotransmitters, involved in advanced neuronal functions (e.g., learning, memory, language, etc.), exerting key effects in the pathologic processes of AD. These neurotransmitters could be involved in the damaging procedure of synaptic plasticity like long-term potentiation and long-term depression in $\mathrm{AD}$ subjects or animal models, which in turn may impair some synapse-based higher brain functions such as memory and cognition [53-55]. Moreover, our results detected several pathways pertaining to neuroendocrine activities (i.e., ovarian steroidogenesis and prolactin signaling), cuing endocrine processes for the pathogenesis of $\mathrm{AD}[56,57]$. In addition, the adipocytokine signaling pathway was enriched in Alzgset. Adipocytokines, including leptin, adiponectin, NAMPT, RBP-4, and other proinflammatory cytokines, have attracted much attention due to their close connection with $\mathrm{AD}[32,57,58]$. Detection of the adipocytokine signaling pathway in this study provides further evidence for the relationship between adipocytokine and the development and progression of $\mathrm{AD}$, and may also support the idea that AD could be a metabolic disease [59-61]. As suggested by the results shown, the molecular mechanisms underlying $\mathrm{AD}$ are pretty complicated, calling for further thorough studies to decode the underlying pathologic mechanisms.

Of significance, we detected three major pathway groups through pathway crosstalk analysis. One group basically involved the pathways related to the nervous system and metabolism-related activities. Amid these pathways, cholinergic synapse, the calcium signaling pathway, dopaminergic synapse, serotonergic synapse, and neurotrophin signaling have been well dissected to 
function in the progress of $\mathrm{AD}$ [62-65]. In the second module, pathways were largely dominated by immune response or related functions, and by cell growth/survival and neuroendocrine pathways for the third group. Furthermore, we could notice that these three pathway modules were interconnected and acted as an immuneendocrine-neuronal regulatory network for the AD-related pathological conditions. Of note, one pathway (i.e., intestinal immune network for IgA production) was found to be a component part of the immune module. These results might suggest that the gut-brain axis, made up of immune, neuroendocrine, and neuronal components, was involved in the pathogenesis of $\mathrm{AD}$ [66-68], in line with results from pathway crosstalk analysis (i.e., there being three similar modules containing Alzgset-enriched pathways). Subsequently, via in-depth examination, we observed that the immune module has plenty of pathway crosstalks and plenty of crosstalk strength. In turn, the cell growth/survival and neuroendocrine module has lower number and less strength, compared with the immune module; however, in terms of the neural module, the number and strength of crosstalks are greater and larger. In spite of the limited number of crosstalks, there exist paramount crosstalk levels among metabolic pathways. These observed results might provide causal and regulatory hints for $\mathrm{AD}$. Integrating results from biochemical pathway and pathway crosstalk analyses and the a-priori biological knowledge base, the major pathways related to AD could be summarized in a diagram (Fig. 3).
Further, we extracted an AD-specific protein network on the basis of the human protein-protein interaction network. It is worth noting that some linking genes outside Alzgset but included in the human protein-protein interaction network may be potentially related to AD. For example, nuclear respiratory factor-1 (NRF1) could be affected by early changes in genes participating in the insulin and energy metabolism pathways in an APP/PS1 transgenic mouse model of AD [69]. TYROBP, a transmembrane signaling protein, appeared in our $\mathrm{AD}$-specific subnetwork. By constructing gene regulatory networks in 1647 postmortem brain tissues from late-onset Alzheimer's disease (LOAD) patients and normal subjects, an immune and microglia-related module dominated by genes participating in pathogen phagocytosis was identified, with $T Y R O B P$ as a key causal regulator upregulated in LOAD [70]. $C D H 2$, a classical cadherin playing roles in the development of the nervous system, was found with the pathogenic copy number variations from 261 early-onset familial Alzheimer's disease and early/mixed-onset pedigree individuals using high-density DNA microarrays [71]. By applying cell-based studies and $F B X O 2$ knockout mice, it was found that $F B X O 2$ could regulate amyloid precursor protein-related activities in the brain and might modulate $\mathrm{AD}$ pathogenesis, coupling with our result to consolidate its involvement in AD [72]. Although no evidence indicated that VSTM2L, one of the intermediate genes, was directly related to $\mathrm{AD}$, it

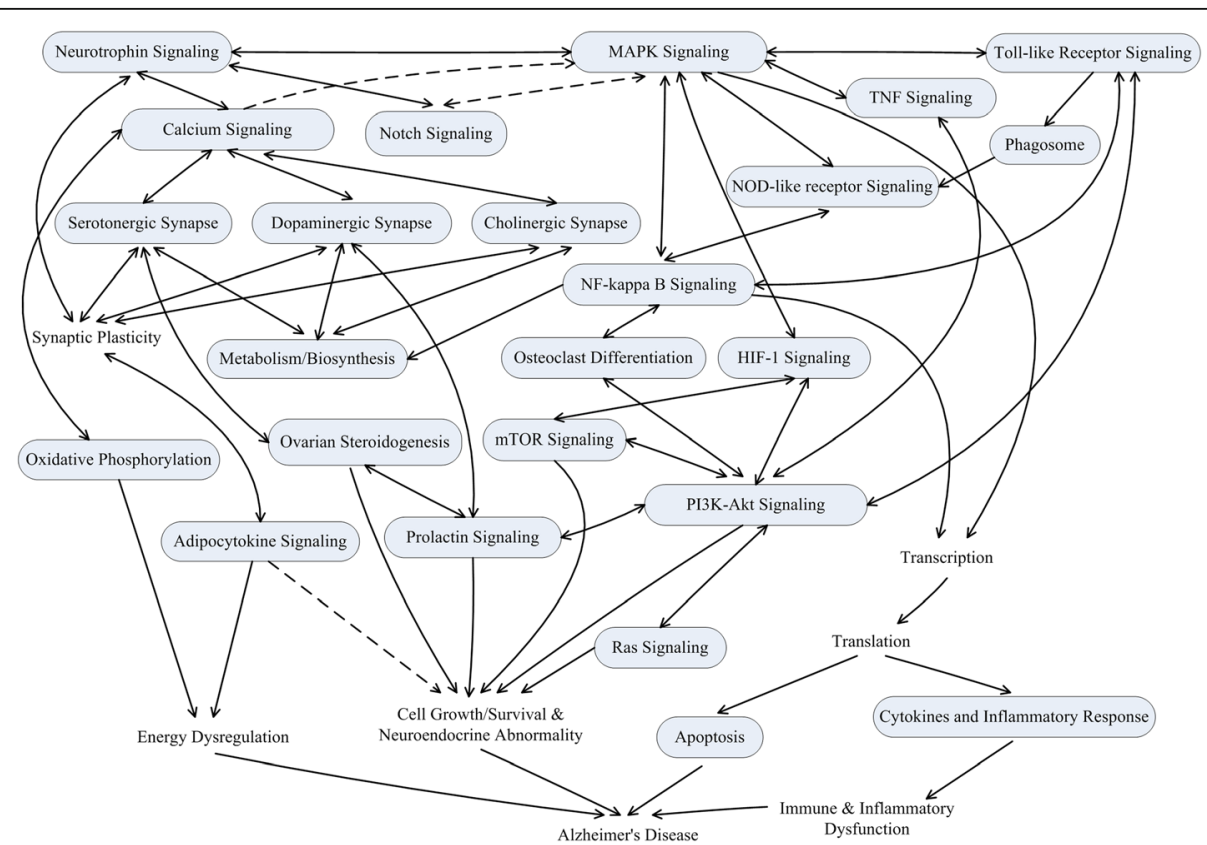

Fig. 3 Main biochemical pathways related to AD. Numbers of genetics-based studies have revealed the fact that AD is actually a complex disorder. These major biochemical pathways involved in AD were connected based on their biological relations 
interacted with ataxin 1 (ATXN1) of Alzgset [73], whose biological function is presently unknown, and also might be a secreted antagonist of Humanin $(H N)$ [74] which mediated attenuation of AD-related memory impairment and $\mathrm{A} \beta$-induced $\mathrm{AD}$-like pathological changes $[75,76]$. As specified by the results detailed, this protein subnetwork predicting approach could not only engender a significant predicted subnetwork of Alzgset for $\mathrm{AD}$, but could also possess the potentiality to detect promising relevant genes.

There have been several available datasets or projects focused on the curation of AD-related genes, including AlzGene [77], Alzheimer's Disease Neuroimaging Initiative (ADNI) [78], the Alzheimer Disease \& Frontotemporal Dementia Mutation Database (AD\&FTDMDB) [79], and AlzBase [80]. While AlzGene maintains a comprehensive catalog of genetic association studies on $\mathrm{AD}$ and also includes results from meta-analysis of polymorphisms with genotype data available in several GWAS projects on $\mathrm{AD}$, AD\&FTDMDB is dedicated to the known mutations of genes associated with $\mathrm{AD}$ and frontotemporal dementias from the published reports or presentations at scientific meetings. The ADNI project aims at facilitating the investigation of genetic influences on $\mathrm{AD}$ onset and progression reflected in imaging changes, fluid biomarkers, and cognitive status. It has reported several neuroimaging GWAS with imaging quotas as quantitative phenotypes, such as hippocampal volume and hippocampal gray matter density. On the other hand, AlzBase is an integrative database for genes dysregulated in $\mathrm{AD}$ and related diseases, and comprises annotations and expression information on more than 7800 differentially expressed genes collected from multiple microarray datasets. These datasets with different features provide valuable information on genes and/or phenotypes for exploring and understanding $\mathrm{AD}$ and its mechanisms.

Similar to AlzGene, Alzgset is also a compilation of ADrelated genes identified in genetic association studies. While AlzGene includes both genes showing positive and negative association with $\mathrm{AD}$, Alzgset focuses only on the genes reported to be positively associated with $\mathrm{AD}$ by the original authors. Because AlzGene has not been updated since April 2011, results from many recent genetic association studies may not be included. In association with studies on candidate genes, some genes may each possess a mild to moderate $p$ value, but two or more genes could collectively show a more significant association with $\mathrm{AD}$ due to the fact they probably act in a concerted manner. In such cases, all of these candidates were included in Alzgset as long as the original authors could provide sufficient evidence. On the other hand, the genes in AlzGene were selected from meta-analyses for each polymorphism and a relative uniform criterion was adopted, so the genes mentioned may be neglected. Thus, Alzgset should offer an informative supplement for AlzGene and serve as a useful dataset for $\mathrm{AD}$ investigation.

However, there were several limitations in this study. First, our pathway-based and network-based analyses results relied on genes in the publications reported to be associated with $\mathrm{AD}$. In view of the fact that identification of risk genes for AD is still an ongoing task, the GO biological process terms, biochemical pathways, and results derived from network analysis should also be treated in the similar manner. Second, we adopted the results and conclusions offered by the original authors of each selected report when collecting the genes, which inevitably impacts our results due to possible bias and insufficiency in the available reports. Then, in order to decrease the false-positive rate of $\mathrm{AD}$ associated genes, we eliminated reports with insignificant or negative results. Nevertheless, we cannot avoid the fact that some genes in those studies might be actually associated with the disease phenotype. Additionally, although the GO terms enriched in Alzgset could provide valuable hints and might serve as an important resource for understanding the molecular mechanisms of $\mathrm{AD}$, it should be noted that GO is biased towards fields like cancer biology and the concepts related to neurology are underrepresented [81]. Thus, some important neurological processes related to AD may be missed in our analysis. At the same time, despite overall levels of protein-protein interaction databases having been greatly improved, the present human interactome is still incomplete and some false-positive data may also be included [82]. Thus, the present research status of the human interactome may also influence our results. It can be expected that, as the protein-protein interaction data become more comprehensive and accurate, the inferred AD-specific subnetwork can become more reliable and valuable.

\section{Conclusions}

In summary, via a systems biology approach, we investigated the pathways and molecular networks related to $\mathrm{AD}$ based on the genes associated with the disease. Integrating biological function, biochemical pathway, and pathway crosstalk analyses, we identified that biochemical processes and pathways linked with lipid and/or lipoprotein-related processes, metabolism, the immune system, and neural development were overrepresented among Alzgset and there existed three interconnected pathway modules: neuronal and metabolic module, cell growth/survival and neuroendocrine clique, and immunological cluster. What is more, an AD-specific protein network was built via the Steiner minimal tree algorithm and some novel genes latently associated with AD were predicted. Such analysis of genes involved in $\mathrm{AD}$ will not only improve our understanding of the 
contribution of genetic factors and their interaction with environmental factors to the pathogenesis of this disease, but will also help us to identify potential biomarkers for further exploration. It could be anticipated that as more genetic factors related to $\mathrm{AD}$ are identified, a systematic and comprehensive analysis such as the one adopted in this study will be more useful to explore the molecular mechanisms underlying $\mathrm{AD}$.

\section{Additional files}

Additional file 1: Is a list of the human interactome utilized in this study. The human protein interaction network contains 16,022 genes/ proteins and 228,122 interactions. (TXT 5293 kb)

Additional file 2: Table S1. Is presenting a list of genes associated with Alzheimer's disease and Table S2 presenting the GO biological process terms enriched in Alzgset. (DOC $990 \mathrm{~kb}$ )

\section{Abbreviations}

AD: Alzheimer's disease; Alzgset: Alzheimer's disease gene set; APOE: Apolipoprotein E; APP: Amyloid precursor protein; DYRK1A: Dual specificity tyrosine-phosphorylation-regulated kinase 1A; FDR: False discovery rate; GO: Gene Ontology; GSK3B: Glycogen synthase kinase 3 beta; GWAS: Genome-wide association study; JC: Jaccard coefficient; OC: Overlap coefficient; PESN1: Presenilin 1; PINA: Protein interaction network analysis; PPI: Protein-protein interaction

\section{Acknowledgements}

The authors thank Dr Tao Zhang, Dr Xianfu Yi and Dr Haixuan Qiao for helpful discussions in preparation of the manuscript.

\section{Funding}

This project was supported in part by grants from the National Key Research and Development Program of China (No. 2016YFC0906300), the National Natural Science Foundation of China (No. 31271411 and No. 61202379), and the Scientific Research Foundation for the Returned Overseas Chinese Scholars, State Education Ministry of China. The funding bodies played no role in the design of the study and collection, analysis, and interpretation of data and in writing the manuscript.

\section{Availability of data and materials}

Provided as additional supporting files.

\section{Authors' contributions}

Y-SH, JX, LZ, and JW designed the experiments. Y-SH, JX, YH, and JW performed the experiments and data analysis. Y-SH, LZ, and JW wrote the manuscript. All authors read and approved the final manuscript.

\section{Authors' information}

Y-SH, JX, YH, and JW are from the School of Biomedical Engineering, Tianjin Medical University, Tianjin, China. LZ is from the School of Computer Science and Technology, Tianjin University, Tianjin, China.

\section{Competing interests}

The authors declare that they have no competing interests.

\section{Consent for publication}

Not applicable.

\section{Ethics approval and consent to participate}

Not applicable.

\section{Publisher's Note}

Springer Nature remains neutral with regard to jurisdictional claims in published maps and institutional affiliations.
Received: 12 October 2016 Accepted: 1 March 2017

Published online: 27 April 2017

\section{References}

1. Ballard C, Gauthier S, Corbett A, Brayne C, Aarsland D, Jones E. Alzheimer's disease. Lancet. 2011;377(9770):1019-31.

2. Burns A, lliffe S. Alzheimer's disease. BMJ. 2009;338:b158.

3. Lozano R, Naghavi M, Foreman K, Lim S, Shibuya K, Aboyans V, Abraham J, Adair T, Aggarwal R, Ahn SY, et al. Global and regional mortality from 235 causes of death for 20 age groups in 1990 and 2010: a systematic analysis for the Global Burden of Disease Study 2010. Lancet. 2012:380(9859):2095-128.

4. Reitz C, Mayeux R. Alzheimer disease: epidemiology, diagnostic criteria, risk factors and biomarkers. Biochem Pharmacol. 2014;88(4):640-51.

5. Ager RR, Davis JL, Agazaryan A, Benavente F, Poon WW, LaFerla FM, BlurtonJones M. Human neural stem cells improve cognition and promote synaptic growth in two complementary transgenic models of Alzheimer's disease and neuronal loss. Hippocampus. 2015;25(7):813-26.

6. Bateman RJ, Xiong C, Benzinger TL, Fagan AM, Goate A, Fox NC, Marcus DS, Cairns NJ, Xie X, Blazey TM, et al. Clinical and biomarker changes in dominantly inherited Alzheimer's disease. N Engl J Med. 2012;367(9):795-804

7. Solomon A, Mangialasche F, Richard E, Andrieu S, Bennett DA, Breteler M, Fratiglioni L, Hooshmand B, Khachaturian AS, Schneider LS, et al. Advances in the prevention of Alzheimer's disease and dementia. J Intern Med. 2014;275(3):229-50.

8. Ryu JK, Cho T, Choi HB, Jantaratnotai N, McLarnon JG. Pharmacological antagonism of interleukin-8 receptor CXCR2 inhibits inflammatory reactivity and is neuroprotective in an animal model of Alzheimer's disease. J Neuroinflammation. 2015;12:144.

9. Allen M, Zou F, Chai HS, Younkin CS, Crook J, Pankratz VS, Carrasquillo MM, Rowley CN, Nair AA, Middha S, et al. Novel late-onset Alzheimer disease loci variants associate with brain gene expression. Neurology. 2012;79(3):221-8.

10. Naj AC, Jun G, Reitz C, Kunkle BW, Perry W, Park YS, Beecham GW Rajbhandary RA, Hamilton-Nelson KL, Wang LS, et al. Effects of multiple genetic loci on age at onset in late-onset Alzheimer disease: a genomewide association study. JAMA Neurol. 2014;71(11):1394-404.

11. Cabral C, Morgado PM, Campos Costa D, Silveira M. Alzheimers Disease Neuroimaging Initiative. Predicting conversion from MCI to AD with FDG-PET brain images at different prodromal stages. Comput Biol Med. 2015;58:101-9.

12. Gatz M, Reynolds CA, Fratiglioni L, Johansson B, Mortimer JA, Berg S, Fiske A, Pedersen NL. Role of genes and environments for explaining Alzheimer disease. Arch Gen Psychiatry. 2006:63(2):168-74.

13. Ertekin-Taner N. Genetics of Alzheimer disease in the pre- and post-GWAS era. Alzheimers Res Ther. 2010;2(1):3.

14. Zhang B, Kirov S, Snoddy J. WebGestalt: an integrated system for exploring gene sets in various biological contexts. Nucleic Acids Res. 2005;33(Web Server issue):W741-8.

15. Chen J, Bardes EE, Aronow BJ, Jegga AG. ToppGene Suite for gene list enrichment analysis and candidate gene prioritization. Nucleic Acids Res. 2009;37(Web Server issue):W305-11.

16. Shannon P, Markiel A, Ozier O, Baliga NS, Wang JT, Ramage D, Amin N, Schwikowski B, Ideker T. Cytoscape: a software environment for integrated models of biomolecular interaction networks. Genome Res. 2003;13(11): 2498-504.

17. Cowley MJ, Pinese M, Kassahn KS, Waddell N, Pearson JV, Grimmond SM, Biankin AV, Hautaniemi S, Wu J. PINA v2.0: mining interactome modules. Nucleic Acids Res. 2012;40(Database issue):D862-5

18. Menche J, Sharma A, Kitsak M, Ghiassian SD, Vidal M, Loscalzo J, Barabasi AL. Disease networks. Uncovering disease-disease relationships through the incomplete interactome. Science. 2015:347(6224):1257601.

19. Barabasi AL, Gulbahce N, Loscalzo J. Network medicine: a network-based approach to human disease. Nat Rev Genet. 2011;12(1):56-68.

20. Klein P, Ravi R. A nearly best-possible approximation algorithm for nodeweighted Steiner trees. J Algorithms. 1995;19(1):104-15.

21. Zheng S, Zhao Z. GenRev: exploring functional relevance of genes in molecular networks. Genomics. 2012;99(3):183-8.

22. Erdos $P$, Rényi A. On the evolution of random graphs. Publ Math Inst Hungar Acad Sci. 1960;5:17-61.

23. Roussotte FF, Daianu M, Jahanshad N, Leonardo CD, Thompson PM Neuroimaging and genetic risk for Alzheimer's disease and addictionrelated degenerative brain disorders. Brain Imaging Behav. 2014;8(2): 217-33. 
24. Anstey KJ, Mack HA, Cherbuin N. Alcohol consumption as a risk factor for dementia and cognitive decline: meta-analysis of prospective studies. Am J Geriatr Psychiatry. 2009;17(7):542-55.

25. Gjoneska E, Pfenning AR, Mathys H, Quon G, Kundaje A, Tsai LH, Kellis M Conserved epigenomic signals in mice and humans reveal immune basis of Alzheimer's disease. Nature. 2015:518(7539):365-9.

26. Heneka MT, Golenbock DT, Latz E. Innate immunity in Alzheimer's disease. Nat Immunol. 2015;16(3):229-36.

27. Wang C, Zhang X, Teng Z, Zhang T, Li Y. Downregulation of PI3K/Akt/mTOR signaling pathway in curcumin-induced autophagy in APP/PS1 double transgenic mice. Eur J Pharmacol. 2014;740:312-20.

28. Polychronidou E, Vlachakis D, Vlamos P, Baumann M, Kossida S. Notch signaling and ageing. Adv Exp Med Biol. 2015;822:25-36.

29. Wang D, Ho L, Faith J, Ono K, Janle EM, Lachcik PJ, Cooper BR, Jannasch AH, D'Arcy BR, Williams BA, et al. Role of intestinal microbiota in the generation of polyphenol-derived phenolic acid mediated attenuation of Alzheimer's disease beta-amyloid oligomerization. Mol Nutr Food Res. 2015;59(6):1025-40.

30. Alam MZ, Alam Q, Kamal MA, Abuzenadah AM, Haque A. A possible link of gut microbiota alteration in type 2 diabetes and Alzheimer's disease pathogenicity: an update. CNS Neurol Disord Drug Targets. 2014;13(3):383-90.

31. Roos PM. Osteoporosis in neurodegeneration. J Trace Elem Med Biol. 2014;28(4):418-21.

32. Letra L, Santana I, Seica R. Obesity as a risk factor for Alzheimer's disease: the role of adipocytokines. Metab Brain Dis. 2014;29(3):563-8.

33. Teixeira AL, Diniz BS, Campos AC, Miranda AS, Rocha NP, Talib LL, Gattaz WF, Forlenza OV. Decreased levels of circulating adiponectin in mild cognitive impairment and Alzheimer's disease. Neruomol Med. 2013;15(1):115-21.

34. Jia P, Kao CF, Kuo PH, Zhao Z. A comprehensive network and pathway analysis of candidate genes in major depressive disorder. BMC Syst Biol. 2011;5 Suppl 3:S12.

35. Leal MC, Magnani N, Villordo S, Buslje CM, Evelson P, Castano EM, Morelli L. Transcriptional regulation of insulin-degrading enzyme modulates mitochondrial amyloid beta (Abeta) peptide catabolism and functionality. J Biol Chem. 2013;288(18):12920-31.

36. Conejero-Goldberg C, Hyde TM, Chen S, Dreses-Werringloer U, Herman MM, Kleinman JE, Davies P, Goldberg TE. Molecular signatures in post-mortem brain tissue of younger individuals at high risk for Alzheimer's disease as based on APOE genotype. Mol Psychiatry. 2011;16(8):836-47.

37. Adori C, Gluck L, Barde S, Yoshitake T, Kovacs GG, Mulder J, Magloczky Z, Havas L, Bolcskei K, Mitsios N, et al. Critical role of somatostatin receptor 2 in the vulnerability of the central noradrenergic system: new aspects on Alzheimer's disease. Acta Neuropathol. 2015;129(4):541-63.

38. Capsoni S, Amato G, Vignone D, Criscuolo C, Nykjaer A, Cattaneo A. Dissecting the role of sortilin receptor signaling in neurodegeneration induced by NGF deprivation. Biochem Biophys Res Commun. 2013;431(3):579-85.

39. Satoh K, Abe-Dohmae S, Yokoyama S, St George-Hyslop P, Fraser PE. ATP-binding cassette transporter A7 (ABCA7) loss of function alters Alzheimer amyloid processing. J Biol Chem. 2015;290(40):24152-65.

40. Inkster B, Rao AW, Ridler K, Filippini N, Whitcher B, Nichols TE, Wetten S, Gibson RA, Borrie M, Kertesz A, et al. Genetic variation in GOLM1 and prefrontal cortical volume in Alzheimer's disease. Neurobiol Aging. 2012;33(3):457-65.

41. Raha AA, Vaishnav RA, Friedland RP, Bomford A, Raha-Chowdhury R. The systemic iron-regulatory proteins hepcidin and ferroportin are reduced in the brain in Alzheimer's disease. Acta Neuropathol Commun. 2013;1:55.

42. Kong W, Zhang J, Mou X, Yang Y. Integrating gene expression and protein interaction data for signaling pathway prediction of Alzheimer's disease. Comput Math Methods Med. 2014;2014:340758.

43. Ponzoni I, Nueda M, Tarazona S, Gotz S, Montaner D, Dussaut J, Dopazo J, Conesa A. Pathway network inference from gene expression data. BMC Syst Biol. 2014;8 Suppl 2:S7.

44. Sun Y, Bresell A, Rantalainen M, Hoglund K, Lebouvier T, Salter H. Alzheimer Disease Neuroimaging Initiative. An integrated bioinformatics approach for identifying genetic markers that predict cerebrospinal fluid biomarker p-tau181/Abeta1-42 ratio in ApoE4-negative mild cognitive impairment patients. J Alzheimers Dis. 2015;45(4):1061-76.

45. Williams-Skipp C, Raman T, Valuck RJ, Watkins H, Palmer BE, Scheinman RI. Unmasking of a protective tumor necrosis factor receptor I-mediated signal in the collagen-induced arthritis model. Arthritis Rheum. 2009;60(2):408-18.

46. Parra MA, Saarimaki H, Bastin ME, Londono AC, Pettit L, Lopera F, Della Sala S, Abrahams S. Memory binding and white matter integrity in familial Alzheimer's disease. Brain. 2015;138(Pt 5):1355-69.
47. Ahmadian-Attari MM, Dargahi L, Mosaddegh M, Kamalinejad M, Khallaghi B, Noorbala F, Ahmadiani A. Impairment of rat spatial learning and memory in a new model of cold water-induced chronic hypothermia: implication for Alzheimer's disease. Neurotox Res. 2015;28(2):95-107.

48. Peter-Derex L, Yammine P, Bastuji H, Croisile B. Sleep and Alzheimer's disease. Sleep Med Rev. 2015;19:29-38.

49. Suzuki C, Yokote Y, Takahashi T. Changes in daily cognition and behavior of Alzheimer's patients over time: a three-year evaluation using a daily cognition and behavior for Alzheimer's disease scale. Dementia. 2015;14(1):126-35.

50. Satoh J, Tabunoki H, Ishida T, Saito Y, Arima K. Accumulation of a repulsive axonal guidance molecule RGMa in amyloid plaques: a possible hallmark of regenerative failure in Alzheimer's disease brains. Neuropathol Appl Neurobiol. 2013;39(2):109-20.

51. Landlinger C, Oberleitner L, Gruber P, Noiges B, Yatsyk K, Santic R, Mandler M, Staffler G. Active immunization against complement factor C5a: a new therapeutic approach for Alzheimer's disease. J Neuroinflammation. 2015;12:150.

52. Alcolea D, Martinez-Lage P, Sanchez-Juan P, Olazaran J, Antunez C, Izagirre A, Ecay-Torres M, Estanga A, Clerigue M, Guisasola MC, et al. Amyloid precursor protein metabolism and inflammation markers in preclinical Alzheimer disease. Neurology. 2015;85(7):626-33.

53. Wang X, Hu X, Yang Y, Takata T, Sakurai T. Systemic pyruvate administration markedly reduces neuronal death and cognitive impairment in a rat model of Alzheimer's disease. Exp Neurol. 2015;271:145-54.

54. Ahmed T, Blum D, Burnouf S, Demeyer D, Buee-Scherrer V, D'Hooge R, Buee $L$, Balschun D. Rescue of impaired late-phase long-term depression in a tau transgenic mouse model. Neurobiol Aging. 2015;36(2):730-9.

55. Koch G, Di Lorenzo F, Bonni S, Ponzo V, Caltagirone C, Martorana A. Impaired LTP- but not LTD-like cortical plasticity in Alzheimer's disease patients. J Alzheimers Dis. 2012;31(3):593-9.

56. Bethea $C L$, Reddy AP. Ovarian steroids regulate gene expression related to DNA repair and neurodegenerative diseases in serotonin neurons of macaques. Mol Psychiatry. 2015;20(12):1565-78.

57. Folch J, Patraca I, Martinez N, Pedros I, Petrov D, Ettcheto M, Abad S, Marin M, Beas-Zarate C, Camins A. The role of leptin in the sporadic form of Alzheimer's disease. Interactions with the adipokines amylin, ghrelin and the pituitary hormone prolactin. Life Sci. 2015;140:19-28.

58. Magalhaes CA, Carvalho MG, Sousa LP, Caramelli P, Gomes KB. Leptin in Alzheimer's disease. Clin Chim Acta. 2015;450:162-8.

59. de la Monte SM, Tong M. Brain metabolic dysfunction at the core of Alzheimer's disease. Biochem Pharmacol. 2014;88(4):548-59.

60. Merlo S, Spampinato S, Canonico PL, Copani A, Sortino MA. Alzheimer's disease: brain expression of a metabolic disorder? Trends Endocrinol Metab. 2010;21(9):537-44.

61. Demetrius LA, Driver J. Alzheimer's as a metabolic disease. Biogerontology. 2013;14(6):641-9.

62. Perez SE, He B, Nadeem M, Wuu J, Scheff SW, Abrahamson EE, Ikonomovic MD, Mufson EJ. Resilience of precuneus neurotrophic signaling pathways despite amyloid pathology in prodromal Alzheimer's disease. Biol Psychiatry. 2015;77(8):693-703.

63. Potter PE, Rauschkolb PK, Pandya Y, Sue LI, Sabbagh MN, Walker DG, Beach TG. Pre- and post-synaptic cortical cholinergic deficits are proportional to amyloid plaque presence and density at preclinical stages of Alzheimer's disease. Acta Neuropathol. 2011;122(1):49-60.

64. Pimenova AA, Thathiah A, De Strooper B, Tesseur I. Regulation of amyloid precursor protein processing by serotonin signaling. PLoS One. 2014;9(1):e87014.

65. Egorova P, Popugaeva E, Bezprozvanny I. Disturbed calcium signaling in spinocerebellar ataxias and Alzheimer's disease. Semin Cell Dev Biol. 2015;40:127-33.

66. Scheperjans F. Can microbiota research change our understanding of neurodegenerative diseases? Neurodegener Dis Manag. 2016;6(2):81-5.

67. Ghaisas S, Maher J, Kanthasamy A. Gut microbiome in health and disease: linking the microbiome-gut-brain axis and environmental factors in the pathogenesis of systemic and neurodegenerative diseases. Pharmacol Ther. 2016;158:52-62.

68. Catanzaro R, Anzalone M, Calabrese F, Milazzo M, Capuana M, Italia A, Occhipinti S, Marotta F. The gut microbiota and its correlations with the central nervous system disorders. Panminerva Med. 2015;57(3):127-43.

69. Pedros I, Petrov D, Allgaier M, Sureda F, Barroso E, Beas-Zarate C, Auladell C, Pallas M, Vazquez-Carrera M, Casadesus G, et al. Early alterations in energy metabolism in the hippocampus of APPswe/PS1dE9 mouse model of Alzheimer's disease. Biochim Biophys Acta. 2014;1842(9):1556-66. 
70. Zhang B, Gaiteri C, Bodea LG, Wang Z, McElwee J, Podtelezhnikov AA, Zhang C, Xie T, Tran L, Dobrin R, et al. Integrated systems approach identifies genetic nodes and networks in late-onset Alzheimer's disease. Cell. 2013;153(3):707-20.

71. Hooli BV, Kovacs-Vajna ZM, Mullin K, Blumenthal MA, Mattheisen M, Zhang C, Lange C, Mohapatra G, Bertram L, Tanzi RE. Rare autosomal copy number variations in early-onset familial Alzheimer's disease. Mol Psychiatry. 2014;19(6):676-81.

72. Atkin G, Hunt J, Minakawa E, Sharkey L, Tipper N, Tennant W, Paulson HL. F-box only protein 2 (Fbxo2) regulates amyloid precursor protein levels and processing. J Biol Chem. 2014;289(10):7038-48.

73. Lim J, Hao T, Shaw C, Patel AJ, Szabo G, Rual JF, Fisk CJ, Li N, Smolyar A, Hill $\mathrm{DE}$, et al. A protein-protein interaction network for human inherited ataxias and disorders of Purkinje cell degeneration. Cell. 2006;125(4):801-14.

74. Rossini L, Hashimoto Y, Suzuki H, Kurita M, Gianfriddo M, Scali C, Roncarati R, Franceschini D, Pollio G, Trabalzini L, et al. VSTM2L is a novel secreted antagonist of the neuroprotective peptide Humanin. FASEB J. 2011;25(6): 1983-2000.

75. Matsuoka M. Protective effects of Humanin and calmodulin-like skin protein in Alzheimer's disease and broad range of abnormalities. Mol Neurobiol. 2015;51(3):1232-9.

76. Chai GS, Duan DX, Ma RH, Shen JY, Li HL, Ma ZW, Luo Y, Wang L, Qi XH, Wang $Q$, et al. Humanin attenuates Alzheimer-like cognitive deficits and pathological changes induced by amyloid beta-peptide in rats. Neurosc Bull. 2014;30(6):923-35.

77. Bertram L, McQueen MB, Mullin K, Blacker D, Tanzi RE. Systematic metaanalyses of Alzheimer disease genetic association studies: the AlzGene database. Nat Genet. 2007;39(1):17-23.

78. Saykin AJ, Shen L, Foroud TM, Potkin SG, Swaminathan S, Kim S, Risacher SL, Nho K, Huentelman MJ, Craig DW, et al. Alzheimer's Disease Neuroimaging Initiative biomarkers as quantitative phenotypes: Genetics core aims, progress, and plans. Alzheimers Dement. 2010;6(3):265-73.

79. Cruts M, Theuns J, Van Broeckhoven C. Locus-specific mutation databases for neurodegenerative brain diseases. Hum Mutat. 2012;33(9):1340-4.

80. Bai Z, Han G, Xie B, Wang J, Song F, Peng X, Lei H. AlzBase: an integrative database for gene dysregulation in Alzheimer's disease. Mol Neurobiol. 2016;53(1):310-9.

81. Roncaglia P, Martone ME, Hill DP, Berardini TZ, Foulger RE, Imam FT, Drabkin $\mathrm{H}$, Mungall CJ, Lomax J. The Gene Ontology (GO) cellular component ontology: integration with SAO (Subcellular Anatomy Ontology) and other recent developments. J Biomed Semantics. 2013;4(1):20.

82. Ideker T, Sharan R. Protein networks in disease. Genome Res. 2008;18(4): 644-52.

\section{Submit your next manuscript to BioMed Central and we will help you at every step:}

- We accept pre-submission inquiries

- Our selector tool helps you to find the most relevant journal

- We provide round the clock customer support

- Convenient online submission

- Thorough peer review

- Inclusion in PubMed and all major indexing services

- Maximum visibility for your research

Submit your manuscript at www.biomedcentral.com/submit

) Biomed Central 